\title{
Suppression of heat shock protein 27 expression promotes 5-fluorouracil sensitivity in colon cancer cells in a xenograft model
}

\author{
RYOHEI HAYASHI, YOSHIYUKI ISHII, HIROKI OCHIAI, ATSUSHI MATSUNAGA, \\ TAKASHI ENDO, HIROTOSHI HASEGAWA and YUKO KITAGAWA
}

Department of Surgery, School of Medicine, Keio University, Tokyo 160-8582, Japan

Received April 26, 2012; Accepted June 21, 2012

DOI: 10.3892/or.2012.1935

\begin{abstract}
The present study investigates whether the expression levels of heat shock protein 27 (HSP27) in colon cancer cells are associated with 5-fluorouracil (5-FU) sensitivity in a xenograft model, as well as the mechanism responsible for regulating 5-FU sensitivity. HCT116 cells which have a high expression of HSP27 were stably transfected with specific short hairpin RNA (shRNA) in order to suppress HSP27 expression. The association between HSP27 protein expression levels and 5-FU sensitivity was evaluated in a mouse xenograft model. The mRNA expression of 5-FU metabolic enzymes and cell apoptosis were also analyzed in the transfected cells. The suppression of HSP27 protein expression led to enhanced 5-FU sensitivity. The mRNA expression levels of dihydropyrimidine dehydrogenase and orotate phosphoribosyltransferase, but not those of thymidylate synthase, and the number of apoptotic cells increased in the transfected cells after 5-FU exposure. In conclusion, the suppression of HSP27 expression in colon cancer cells may promote 5-FU sensitivity by inducing apoptosis, despite the acceleration in 5-FU metabolism.
\end{abstract}

\section{Introduction}

Over the last few decades, systemic chemotherapy for colon cancer has markedly changed worldwide. Combinations of 5-fluorouracil (5-FU) with other cytotoxic agents, such as oxaliplatin and irinotecan, have improved the prognosis of patients with advanced colon cancer. Furthermore, the addition of molecular-targeted agents, such as anti-VEGF antibody (bevacizumab) and anti-EGFR antibody (cetuximab/panitumumab), have resulted in dramatic improvements in the survival of patients with advanced colon cancer and are currently regarded as first-line chemotherapy regimens (1-4). Although 5-FU is still

Correspondence to: Dr Yoshiyuki Ishii, Department of Surgery, School of Medicine, Keio University, Shinanomachi 35, Shinjuku-ku, Tokyo 160-8582, Japan

E-mail: yishii@a2.keio.jp

Key words: heat shock protein 27, 5-fluorouracil, colon cancer, xenograft model a key drug in these regimens, the inherent or acquired resistance to 5-FU in colon cancer is a critical problem. A number of studies have reported that 5-FU metabolism-related factors, such as thymidylate synthase (TS), folate co-factors, dihydropyrimidine dehydrogenase (DPD) and orotate phosphoribosyltransferase (OPRT), are associated with the response to or toxicity of 5-FU (5-8). However, there are still no reliable biomarkers for the sensitivity or resistance to 5-FU chemotherapy.

Mammalian heat shock proteins (HSPs) have been classified into four main families based on their molecular weights: HSP90, HSP70, HSP60, and small HSPs (15-30 kDa), including HSP27. These proteins are well known as molecular chaperons in protein-protein interactions, as anti-apoptotic proteins and contributors to cell survival $(9,10)$. Many studies have reported that HSP27 expression contributes to the malignant properties of cancer cells, including tumorigenicity, treatment resistance, and apoptosis inhibition (11-15). In colon cancer, certain studies have reported that HSP27 expression participates in the resistance to doxorubicin or irinotecan in vitro $(16,17)$, and HSP27 has recently been considered as a prognostic marker in clinicopathological studies (18-20). Our previous study also indicated that HSP27 expression participates in the degree of resistance to 5-FU, a key drug for the treatment of colorectal cancer, in experiments performed in vitro (21). In the present study, we sought to clarify whether HSP27 can be used as a clinical biomarker for 5-FU chemotherapy or as a treatment target for 5-FU resistance using a xenograft model.

\section{Materials and methods}

Drug, cell lines and cell culture conditions. The anticancer drug, 5-FU, was purchased from Kyowa Hakko Kogyo Co., Ltd. (Tokyo, Japan). The human colon cancer cell line, HCT116, was obtained from the American Type Culture Collection (Rockville, MD). The cells were grown in Roswell Park Memorial Institute (RPMI)-1640 medium (Gibco, MA). Each culture was supplemented with $10 \%$ fetal bovine serum (CSL Ltd., Melbourne, Australia) and $1 \%$ penicillin/streptomycin. The cells were cultured at $37^{\circ} \mathrm{C}$ with $5 \% \mathrm{CO}_{2}$.

Stable transfection with short hairpin RNA (shRNA). An oligonucleotide for a short hairpin RNA targeting the human 
HSP27 site (5'-UAGCCAUGCUCGUCCUGCCUU-3') was designed (21), so as to contain 5'-BamHI and 3'-EcoRI overhangs. The annealed oligonucleotide was then ligated into a linearized U6 promoter-driven vector (pSIREN-DNR-DsRedExpress Vector; Clontech, Mountain View, CA). As the negative control, an oligonucleotide for a scrambled shHSP27 (5'-ACGUAAGGCGCGUAACGGGTT-3') containing 5'-BamHI and 3 '-EcoRI overhangs was also constructed and ligated to the pSIREN vector.

For transfection, HCT116 cells expressing high levels of HSP27 under normal culture conditions were plated in 6 -well plates at a density of $1 \times 10^{6}$ cells per well and allowed to grow overnight. The cells were transfected with a mixture of $5 \mu \mathrm{g}$ of siRNA-encoding plasmids (the HSP27 target sequence plasmid and the negative control plasmid) and $20 \mu \mathrm{l}$ of Lipofectamine 2000 (Invitrogen Life Technologies, Inc., Carlsbad, CA) in $2 \mathrm{ml}$ of serum-free medium, according to the manufacturer's instructions. After incubation at $37^{\circ} \mathrm{C}$ for $6 \mathrm{~h}$, the medium was replaced with fresh growing medium. The transfected cells, containing a red fluorescent protein, were verified by visualization in living cells using fluorescence microscopy. Some of the transfected clones with different levels of HSP27 expression were monoclonally obtained and named HCT116-shRNA/HSP27-1 to -4 . The control cells, HCT116-scramble/HSP27 and HCT116-mock cells, were established in a similar manner.

Quantitative real-time PCR ( $q R T-P C R$ ) analysis of 5-FU metabolic enzymes. Total RNA was extracted from the cells in each group, and qRT-PCR analysis was performed using a fluorescence-based, real-time detection method [ABI PRISM 7900 Sequence Detection System (TaqMan); Applied Biosystems, Foster City, CA]. The mRNA expression of DPD, OPRT and TS was analyzed in the transfected cells that were either treated or not with 5-FU $(1.28 \mu \mathrm{g} / \mathrm{ml})$ in culture medium for $24 \mathrm{~h}$. The enzyme expression levels of the transfected cells were assessed relative to the levels of the gene transcript in the mock transfected cells (interest/ $\beta$-actin). The sequences of the primers and probes were as follows: DPD: 51F (19 bp), AGGACGCAAGG AGGGTTTG; 134R (20 bp), GTCCGCCGAGTCCTTACTGA; probe-71T (29 bp), CAGTGCCTACAGTCTCGAGTCTGCC AGTG. OPRT: 496F (25 bp), TAGTGTTTTGGAAACTG TTGAGGTT; 586R (20 bp), CTTGCCTCCCTGCTCTCTGT; probe-528T (27 bp), TGGCATCAGTGACCTTCAAGCCC TCCT. TS: 764F (18 bp), GCCTCGGTGTGCCTTTCA; 830R (17 bp), CCCGTGATGTGCGCAAT; probe-785T (21 bp), TCGCCAGCTAC GCCCTGCTCA. $\beta$-actin: 592F (18 bp), TGAGCGCGGCTACAGCTT; 651R (22 bp), TCCTTAA TGTCACGCACGATTT; probe-611T (18 bp), ACCACCA CGGCCGAGCGG. The $25-\mu l$ PCR reaction mixture contained $600 \mathrm{nmol} / 1 \mathrm{l}$ of each primer, $200 \mathrm{nmol} / 1$ each of dATP, dCTP and dGTP, $400 \mu \mathrm{mol} / 1$ of dUTP, $5.5 \mu \mathrm{mol} / 1$ of $\mathrm{MgCl}_{2}$ and $1 \mathrm{X}$ TaqMan buffer A containing a reference dye (all reagents were supplied by Applied Biosystems). The PCR conditions were $50^{\circ} \mathrm{C}$ for $10 \mathrm{sec}$ and $95^{\circ} \mathrm{C}$ for $10 \mathrm{~min}$, followed by 42 cycles at $95^{\circ} \mathrm{C}$ for $15 \mathrm{sec}$ and $60^{\circ} \mathrm{C}$ for $1 \mathrm{~min}$.

Western blot analysis. Total cell lysates were extracted with lysis buffer with standard methods. The quantity of cell lysates was determined using a Bio-Rad DC protein assay kit
(Bio-Rad Laboratories, Hercules, CA), and $20 \mathrm{mg}$ of lysates in total were resolved in Ready Gel (Bio-Rad Laboratories) and transferred to an Immuno-Blot ${ }^{\mathrm{TM}}$ polyvinylidene fluoride membrane (Bio-Rad Laboratories). The membrane was blocked in phosphate-buffered saline (PBS) containing 5\% non-fat milk powder for $2 \mathrm{~h}$ at room temperature, then incubated at $4^{\circ} \mathrm{C}$ overnight with 1:2000-diluted anti-human HSP27 mouse monoclonal antibody (G3.1; Lab Vision Corp., Fremont, CA) or 1:5000-diluted anti-human $\beta$-actin mouse monoclonal antibody (AC74; Sigma, St. Louis, MO). The membranes were incubated for $30 \mathrm{~min}$ with a 1:5000-diluted horseradish peroxidaseconjugated anti-mouse immunoglobulin G (IgG) (Promega Corp., Madison, WI). Bound complexes were detected using the ECL-Plus reagent (Amersham Biosciences Corp., Cardiff, UK) according to the manufacturer's instructions. The density of the band was measured using NIH imaging (Scion) software and normalized to $\beta$-actin. Each experiment was performed in triplicate.

Xenograft model. Female nude mice with a BALB/cA genetic background were purchased from CLEA Japan Co. Ltd., Tokyo, Japan. The mice were maintained under specific pathogen-free conditions using an Isorack in our experimental animal center and fed sterile food and water. Six- to eight-week-old mice weighing 20-22 $\mathrm{g}$ were used for the experiments. To analyze the effect of HSP27 knockdown against 5-FU treatment, HCT116mock, HCT116-scramble/HSP27, and HCT116-shRNA/HSP27 cells $\left(3 \times 10^{6}\right.$ cells per injection), which were resuspended in $100 \mu 1$ of PBS, were injected into the subcutaneous tissues of the bilateral dorsum of ether-anesthetized mice using a 1-ml syringe and a 27-gauge tuberculin needle (Terumo Co., Tokyo, Japan). The tumors were measured (length and width) twice a week using a sliding caliper by the same observer. When the inoculated tumors reached $5 \mathrm{~mm}$ in length, 5-FU (200 $\mu \mathrm{l}$ saline solution) was administered intraperitoneally at a dose of $60 \mathrm{mg} / \mathrm{kg}$ on days 1,5 and 9 ( $n=5$ mice per group). As the control, saline $(200 \mu \mathrm{l})$ was also administered intraperitoneally $(\mathrm{n}=5$ mice). The estimated tumor volume (EV) was calculated as $\mathrm{EV}=$ length $\mathrm{x}$ width ${ }^{2} \mathrm{x} 1 / 2 . \mathrm{EV}$ was plotted against the day since 5-FU treatment initiation to derive a xenograft growth curve. All the mice were sacrificed at three weeks after the initial treatment. Tumors were collected and fixed in $4 \%$ paraformaldehyde (Sigma) at room temperature for $24 \mathrm{~h}$ before processing for sectioning and immunohistochemical staining or the preparation of tumor lysates for western blot analysis. All animal studies were conducted in accordance with the institutional guidelines approved by the Animal Care and Use Committee of our university.

Immunohistochemical staining. Anti-human HSP27 rabbit polyclonal antibody (Stressgen Bioreagents Corp., Victoria, $\mathrm{BC}$, Canada) was used for the immunohistochemical staining of the tumors from the mice. Immunohistochemical staining was performed according to the standard streptavidin-biotin peroxidase complex method using a Histofine ${ }^{\mathrm{TM}}$ SAB-PO[M] kit (Nichirei, Tokyo, Japan). Briefly, deparaffinized sections were placed in methanol containing $1 \%$ hydrogen peroxide for $15 \mathrm{~min}$ to block endogenous peroxidase activity. After washing with PBS, the sections were incubated with anti-HSP27 antibody (1:100 diluted in PBS) overnight at $4^{\circ} \mathrm{C}$ in a moist chamber, and then 
incubated with biotinylated goat anti-rabbit IgG and peroxidaseconjugated streptavidin (Stressgen Bioreagents Corp.) for $30 \mathrm{~min}$ each at room temperature. After a final washing with PBS, the sections were immersed in a PBS solution containing $0.02 \%$ 3,3'-diaminobenzidine and $0.1 \%$ hematoxylin and mounted in balsam.

Detection of caspase-3 activities for apoptosis analysis. The transfected cells were treated with or without $5-\mathrm{FU}(1.28 \mu \mathrm{g} / \mathrm{ml})$ in the culture medium for $24 \mathrm{~h}$. The caspase- 3 activities were then measured using an ApoAlert Caspase-3 Colorimetric assay kit (Clontech), according to the manufacturer's instructions. The cells $\left(2 \times 10^{6}\right)$ were centrifuged at $400 \mathrm{x} \mathrm{g}$ for $5 \mathrm{~min}$. After the extraction of the cell lysates using cell lysis buffer, the supernatants were centrifuged for $10 \mathrm{~min}$ at $4^{\circ} \mathrm{C}$ to precipitate the cellular debris and were then incubated with reaction buffer/dithiothreitol (DTT) mix for $30 \mathrm{~min}$ on ice and caspase-3 substrate for $1 \mathrm{~h}$ at $37^{\circ} \mathrm{C}$. The samples were read at $405 \mathrm{~nm}$ on a microplate reader.

Statistical analysis. Each value was expressed as the mean \pm standard deviation. Statistical analysis was performed using the Student's t-test or the Mann-Whitney U test. The regression analysis was performed using the Statistical Package for Social Sciences (SPSS) 13.0J for Windows (SPSS, Chicago, IL). $\mathrm{P}<0.05$ was considered to indicate a statistically significant difference.

\section{Results}

5-FU sensitivity of shRNA/HSP27-transfected cells in vitro. The 5-FU-resistant colon cancer cells, HCT116, were transfected with HSP27 shRNA ligated to the pSIREN-DNR-DsRedExpress vector (shRNA/HSP27-transfected cells), scrambled shRNA ligated to the pSIREN vector as the control (scramble/ HSP27-transfected cells), or an empty vector (mock-transfected cells). Transfected cells with various HSP27 expression levels were obtained by cloning (Fig. 1; shRNA/HSP27-1 to -4). To evaluate the 5-FU sensitivity, the transfected cells were first treated with 5-FU in vitro, and then the $\mathrm{IC}_{50}$ of each transfectant was measured using an MTT assay. A significant correlation between the $\mathrm{HSP} 27$ protein levels and the $\mathrm{IC}_{50}$ of 5-FU was observed (Fig. 2; $\mathrm{R}=0.931, \mathrm{P}=0.0039$ ).

5-FU sensitivity of shRNA/HSP27-transfected cells in xenograft model. To evaluate the 5-FU sensitivity in the xenograft model, the transfected cells were injected into the subcutaneous tissues of the bilateral dorsum of ether-anesthetized mice. When the inoculated tumors reached $5 \mathrm{~mm}$ in length, 5-FU was administered intraperitoneally at a dose of $60 \mathrm{mg} / \mathrm{kg}$ on days 1,5 and 9 ( $\mathrm{n}=5$ mice per group). Saline $(200 \mu \mathrm{l})$ was also administered intraperitoneally in the control group $(\mathrm{n}=5$ mice). The $\mathrm{EV}$ following 5-FU treatment was significantly suppressed in the mice injected with the shRNA/HSP27-transfected cells, compared to those injected with the scramble/HSP27- and mock-transfected cells (Figs. 3 and 4).

Immunohistochemistry of the tumors in the xenograft model showed high expression levels of HSP27 in the mockand scramble/HSP27-transfected cells, but not in the shRNA/ HSP27-transfected cells (Fig. 5).

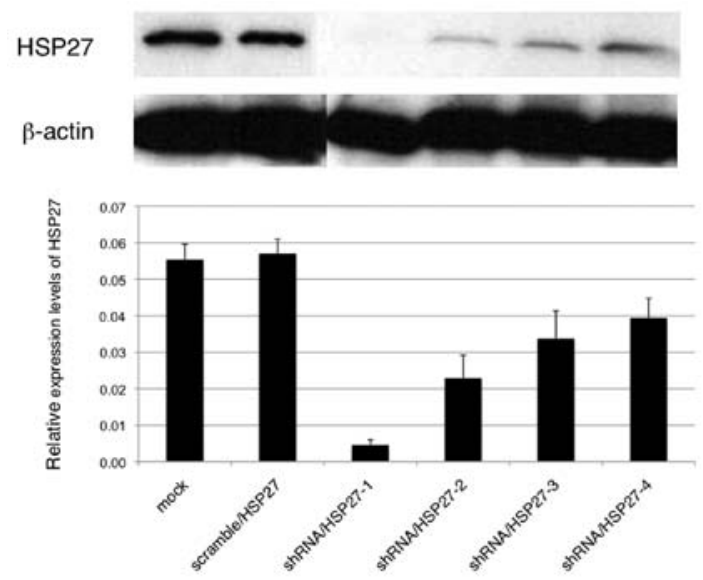

Figure 1. HSP27 protein expression in the shRNA-transfected cells. The 5-FU-resistant colon cancer cells, HCT116, were transfected with shRNA of HSP27. Western blot analysis showed different suppression levels of HSP27 in the transfected cells (shRNA/HSP27-1 to -4). Relative expression levels of HSP27 were assessed by measuring the densities of the bands using NIH image software, and normalized to $\beta$-actin.

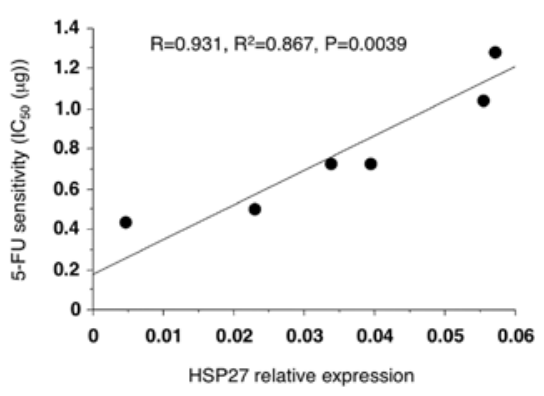

Figure 2. Correlation between HSP27 expression and 5-FU sensitivity $\left(\mathrm{IC}_{50}\right)$ in the shRNA-transfected cells. A significant correlation between HSP27 protein expression and 5-FU sensitivity $\left(\mathrm{IC}_{50}\right)$ by MTT assay was observed in the transfected cells.

Expression of 5-FU metabolic enzymes in shRNA/HSP27transfected cells. To evaluate whether HSP27 expression levels affect the principal 5-FU metabolic enzymes (TS, DPD and OPRT) in the transfected cells treated with 5-FU, a qRT-PCR analysis of 5-FU metabolic enzymes was performed. These enzyme expression levels were assessed relative to the levels of the gene transcript in the mock-transfected cells as the control. Although no significant differences in the mRNA expressions of the three metabolic enzymes were observed between the shRNA/HSP27-transfected cells and the mock- or scramble/ HSP27-transfected cells, the expression of DPD and OPRT was higher in the shRNA/HSP27-transfected cells (Fig. 6). Under the same conditions with 5-FU treatment, apoptosis induction was more frequently observed in the shRNA/HSP27-transfected cells than in the mock- or scramble/HSP27-transfected cells (Fig. 7).

\section{Discussion}

The inherent or acquired resistance to 5-FU-based chemotherapy remains a critical issue in colorectal cancer treatment. Consequently, the identification of biomarkers for chemosensitivity or resistance, such as the $K-R A S$ and $B-R A F$ mutation 

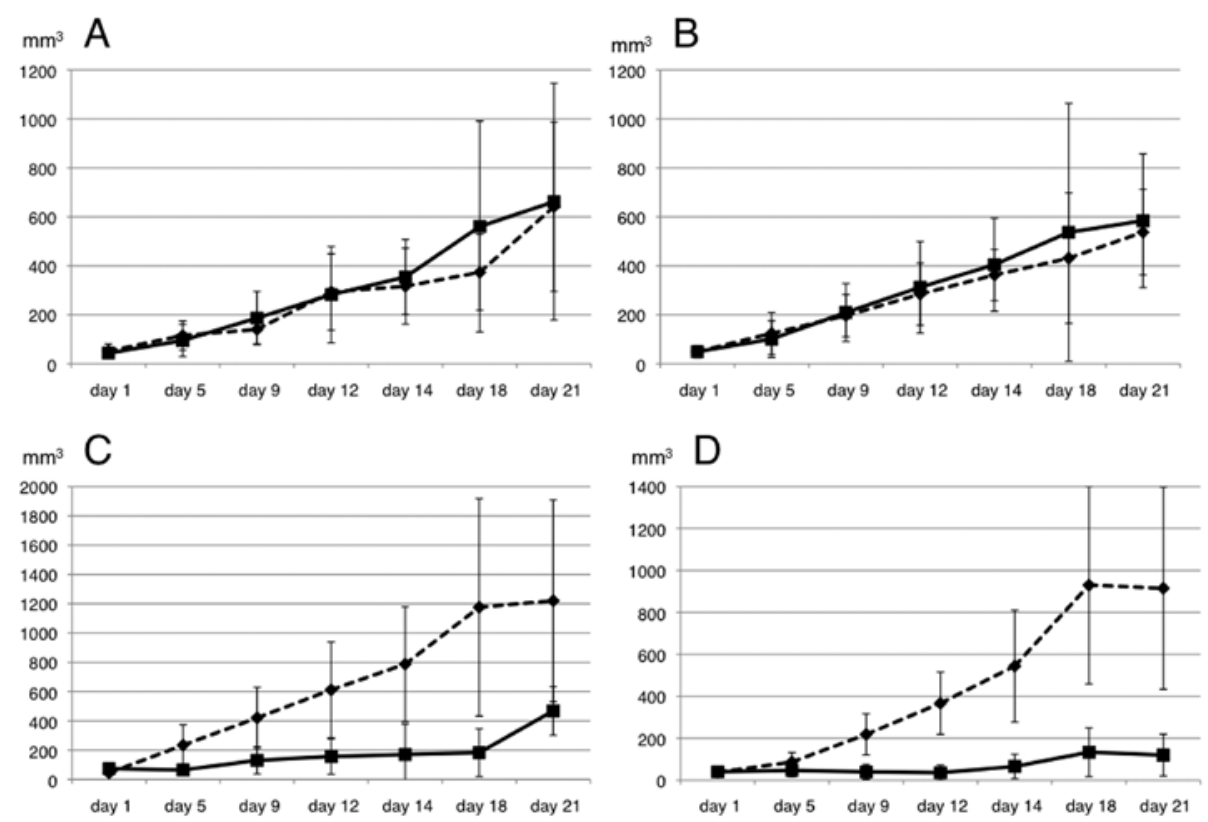

Figure 3. 5-FU sensitivity of the HSP27/shRNA-transfected cells in a xenograft model. Cells $\left(3 \times 10^{6}\right)$ were inoculated in the dorsal subcutaneous tissues of nude mice. When the inoculated tumors reached $5 \mathrm{~mm}$ in length, $5-\mathrm{FU}(60 \mathrm{mg} / \mathrm{kg} / 200 \mathrm{ml}$ saline solution) was administered intraperitoneally on days 1,5 and 9 (black line) Estimated tumor volumes were calculated until 21 days from initial administration of 5-FU. As the control, saline (200 ml) was also administered intraperitoneally (dotted line). (A) Mock-transfected cells. (B) Scramble/HSP27-transfected cells. (C) shRNA/HSP27-1-transfected cells. (D) shRNA/HSP27-2-transfected cells.

A

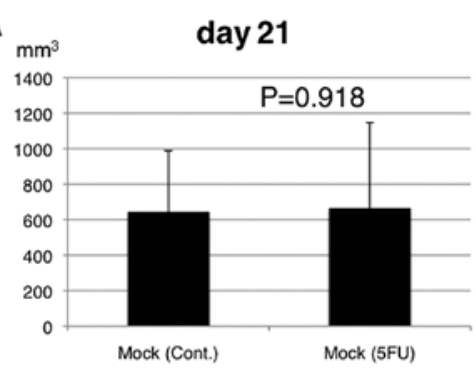

$\mathrm{C}_{\mathrm{mm}^{3}}$

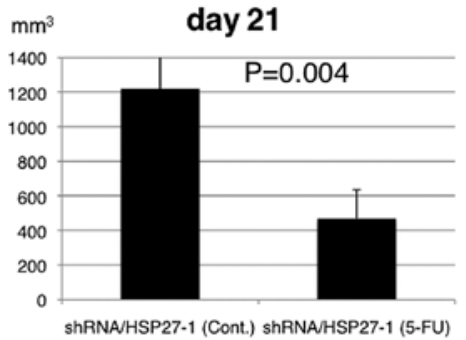

B

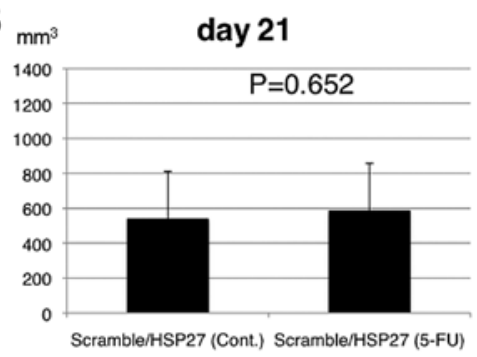

$D_{m^{3}}$ day 21

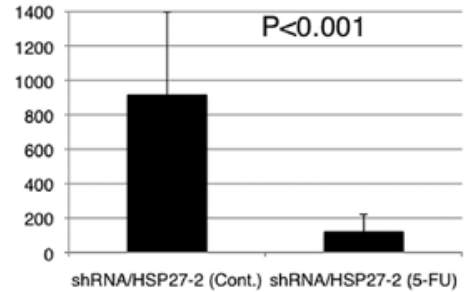

Figure 4. 5-FU sensitivity of the HSP27/shRNA-transfected cells on day 21 in the xenograft model. Estimated tumor volumes were calculated on day 21 after the intraperitoneal injection of 5 -FU. 5-FU $(60 \mathrm{mg} / \mathrm{kg} / 200 \mathrm{ml}$ saline solution) was administered intraperitoneally on days 1,5 and 9 . As the conrol, saline $(200 \mathrm{ml}$ ) was also administered intraperitoneally. (A) Mock-transfected cells. (B) Scramble/HSP27-transfected cells. (C) shRNA/HSP27-1transfected cells. (D) shRNA/HSP27-2-transfected cells.

status for anti-EGFR antibody, is important for personalized chemotherapy and the avoidance of unsuitable chemotherapy and adverse events. In addition, the identification of a new treatment strategy for patients with resistance to chemotherapy is required to improve the survival of colorectal cancer patients.

HSP27 has been widely recognized as a stress-activated, ATP-independent cytoprotective chaperone that is associated with a number of functions, including chemoresistance in several cancers. In colorectal cancer, HSP27 has been reported as a clinical prognostic factor or as a factor of irinotecan resistance in experiments performed in vitro (17-20). Our previous study also reported that the overexpression of HSP27 reduced 5-FU sensitivity and the suppression of HSP27 expression reduced 5-FU resistance in experiments performed in vitro using colon cancer cells (21). Furthermore, in prostate and bladder cancer cells, the inhibition of HSP27 expression reportedly enhanced drug sensitivity in xenograft models (22-24). Thus, HSP27 is considered to be a predictor of cancer prog- 

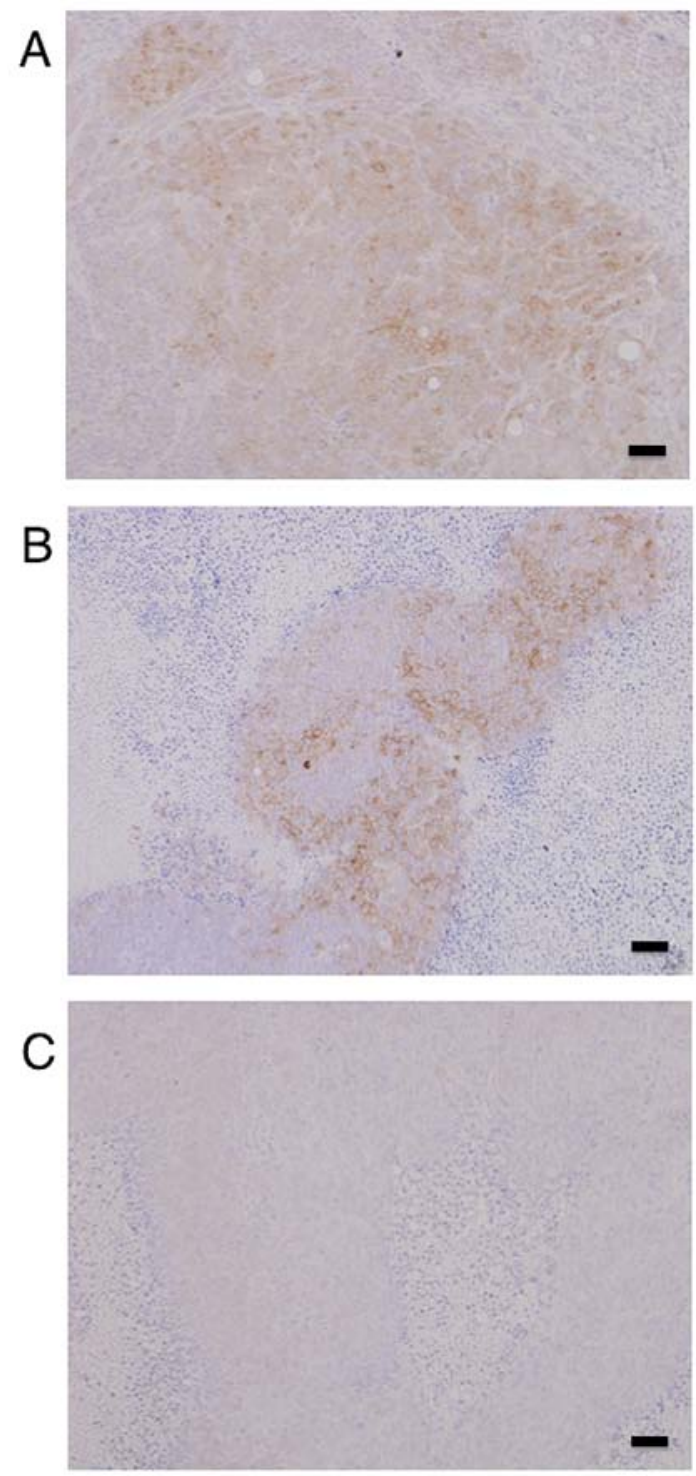

Figure 5. Histology images of the inoculated tumors. Immunohistochemical staining with the anti-HSP27 antibody revealed high expression levels in the mock- and scramble/HSP27-transfected cells, but not in the shRNA/HSP27-1transfected cells. (A) Mock-transfected cells. (B) Scramble/HSP27-transfected cells. (C) shRNA/HSP27-1- transfected cells. Bar, $50 \mathrm{~mm}$.

nosis or a treatment target for several cancers; however, the role of HSP27 in colorectal cancer, including chemoresistance, remains uncertain due to the lack of evidence in xenograft models. Recently, a phase I/II clinical trial of monotherapy using the antisense oligonucleotide (ASO), OGX-427, which inhibits HSP27 expression, in patients with prostate, bladder, ovarian, breast, or non-small cell lung cancer, but not colorectal cancer, was carried out in the United States and Canada, and the therapy proved to be feasible and effective [J Clin Oncol 28 (Suppl): S15, abs. 3077, 2010]. Accordingly, the purpose of this study was to verify that HSP27 can also serve as a target for the treatment of colorectal cancer.

The present study demonstrates that the suppression of HSP27 expression in HSP27 high-expressing colon cancer cells reduces resistance to 5-FU chemotherapy in a xenograft model, and that the induction of apoptosis caused by the suppression of HSP27 expression, which many studies have reported, may be connected

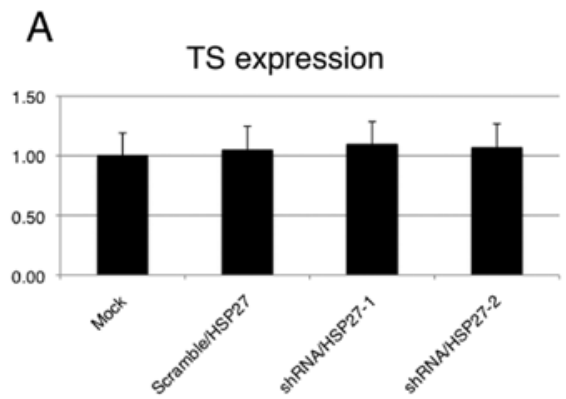

\section{B}

OPRT expression

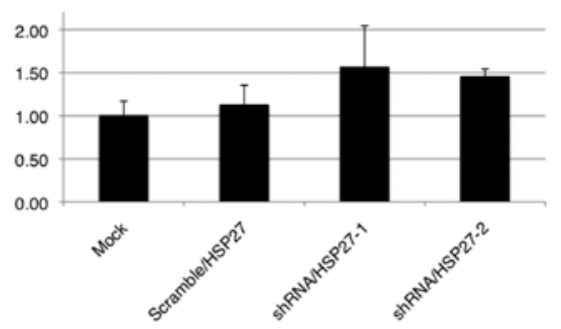

C

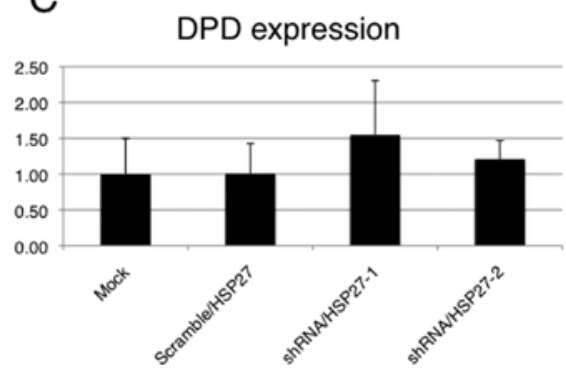

Figure 6. mRNA expression levels of principal 5-FU metabolic enzymes in the shRNA/HSP27-transfected cells. mRNA expression levels of the three enzymes were assessed relative to the levels of the gene transcript in the mocktransfected cells as the control. (A) TS mRNA expression. (B) OPRT mRNA expression. (C) DPD mRNA expression.

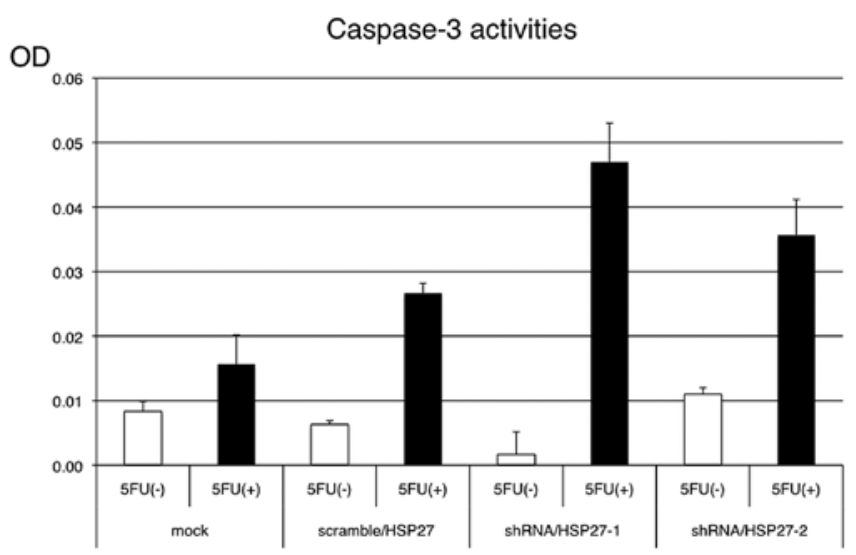

Figure 7. Apoptosis induction by 5-FU treatment in the shRNA/HSP27 transfected cells. Caspase- 3 activities were measured on a microplate reader at $405 \mathrm{~nm}$ using the ApoAlert Caspase-3 Colorimetric assay kit. White bar, no treatment condition as the negative control; black bar, 5-FU treatment condition; OD, optical density.

to 5-FU sensitivity. Our findings are consistent with the findings of several studies that link HSP27 and chemoresistance in 
different cell types. Collectively, this evidence suggests a role of HSP27 in the mediation of 5-FU resistance in colon cancer cells. HSP27 may also serve as a reliable target for the clinical treatment of colon cancer in patients with chemoresistance.

5-FU is well known to induce apoptosis in colon cancer cells, predominantly through the mitochondrial pathway, involving the release of cytochrome $c$ and the subsequent activation of the upstream initiator, caspase-9, and the downstream effector, caspase-3 (25-27). We confirmed that the suppression of HSP27 expression was associated with the induction of apoptosis in colon cancer cells treated with 5-FU. Consequently, HSP27 may function as a negative regulator of the cytochrome $c$-dependent activation of procaspase-3, as previously reported (28). However, whether HSP27 is associated with 5-FU metabolic enzymes, remains unknown. We then analyzed the correlation between HSP27 expression and the principal 5-FU metabolic enzymes, such as TS, DPD and OPRT. The mRNA expression of DPD and OPRT was higher after 5-FU treatment in the shRNA/ HSP27-transfected cells that had a high sensitivity to 5-FU comapred to the control cells, although the difference was not significant. Changes in the TS mRNA expression levels were not clearly observed in the shRNA/HSP27-transfected cells. DPD mediates 5-FU degradation by catabolizing 5-FU to fluoro5,6-dihydrouracil. The enzyme OPRT directly converts 5-FU to 5-fluorouridine-5'- monophosphate and inhibits normal RNA or DNA synthesis in tumor cells. TS is inhibited by 5-fluoro-2'-deoxyuridine-5'- monophosphate (FdUMP) derived from 5-FU, leading to the inhibition of DNA synthesis. Consequently, high TS levels in tumor tissues are considered to be associated with the low efficacy of 5-FU treatment. In consideration of the functions of these enzymes, shRNA/HSP27-transfected colon cancer cells (with a suppressed HSP27 expression) may promote 5-FU metabolism by increasing the expression of DPD and OPRT to remove 5-FU from the cytoplasm, in order to enable cell survival.

In conclusion, this study suggests that the suppression of HSP27 expression in colon cancer cells promotes 5-FU sensitivity by inducing apoptosis, while also accelerating 5-FU metabolism to avoid cell death. Further investigation, such as an HSP27 functional study examining HSP27 phosphorylation activity and signal regulation, may lead to novel treatments for colorectal cancer.

\section{References}

1. Van Cutsem E, Rivera F, Berry S, et al; First BEAT investigators: Safety and efficacy of first-line bevacizumab with FOLFOX XELOX, FOLFIRI and fluoropyrimidines in metastatic colorectal cancer: the BEAT study. Ann Oncol 20: 1842-1847, 2009.

2. Van Cutsem E, Köhne CH, Hitre E, et al: Cetuximab and chemotherapy as initial treatment for metastatic colorectal cancer. $\mathrm{N}$ Engl J Med 360: 1408-1417, 2009.

3. Tol J, Koopman M, Cats A, et al: Chemotherapy, bevacizumab, and cetuximab in metastatic colorectal cancer. $\mathrm{N}$ Engl $\mathbf{J}$ Med 360: 563-572, 2009.

4. Hurwitz H, Fehrenbacher L, Novotny W, et al: Bevacizumab plus irinotecan, fluorouracil, and leucovorin for metastatic colorectal cancer. N Engl J Med 350: 2335-2342, 2004.

5. Kinoshita M, Kodera Y, Hibi K, et al: Gene expression profile of 5-fluorouracil metabolic enzymes in primary colorectal cancer: potential as predictive parameters for response to fluorouracilbased chemotherapy. Anticancer Res 27: 851-856, 2007.

6. Okumura K, Shiomi H, Mekata E, et al: Correlation between chemosensitivity and mRNA expression level of 5-fluorouracilrelated metabolic enzymes during liver metastasis of colorectal cancer. Oncol Rep 15: 875-882, 2006.
7. Aschele C,Debernardis D,Casazza S,et al: Immunohistochemical quantitation of thymidylate synthase expression in colorectal cancer metastases predicts for clinical outcome to fluorouracilbased chemotherapy. J Clin Oncol 17: 1760-1770, 1999.

8. Soong R, Shah N, Salto-Tellez M, et al: Prognostic significance of thymidylate synthase, dihydropyrimidine dehydrogenase and thymidine phosphorylase protein expression in colorectal cancer patients treated with or without 5-fluorouracil-based chemotherapy. Ann Oncol 19: 915-919, 2008.

9. Bruey JM, Ducasse C, Bonniaud P, et al: Hsp27 negatively regulates cell death by interacting with cytochrome c. Nat Cell Biol 2: 645-652, 2000.

10. Mehlen P, Schulze-Osthoff K and Arrigo AP: Small stress proteins as novel regulators of apoptosis. Heat shock protein 27 blocks Fas/ APO-1- and staurosporine-induced cell death. J Biol Chem 271: 16510-16514, 1996

11. Andrieu C, Taieb D, Baylot V, et al: Heat shock protein 27 confers resistance to androgen ablation and chemotherapy in prostate cancer cells through eIF4E. Oncogene 29: 1883-1896, 2010.

12. Sarto C, Valsecchi C, Magni F, et al: Expression of heat shock protein 27 in human renal cell carcinoma. Proteomics 4: 2252-2260, 2004.

13. Song TF, Zhang ZF, Liu L, Yang T, Jiang J and Li P: Small interfering RNA-mediated silencing of heat shock protein 27 (HSP27) increases chemosensitivity to paclitaxel by increasing production of reactive oxygen species in human ovarian cancer cells (HO8910). J Int Med Res 37: 1375-1388, 2009.

14. Ciocca DR, Fuqua SA, Lock-Lim S, Toft DO, Welch WJ and McGuire WL: Response of human breast cancer cells to heat shock and chemotherapeutic drugs. Cancer Res 52: 3648-3654, 1992.

15. Vargas-Roig LM, Gago FE, Tello O, Aznar JC and Ciocca DR: Heat shock protein expression and drug resistance in breast cancer patients treated with induction chemotherapy. Int J Cancer 79: 468-475, 1998.

16. Garrido C, Mehlen P, Fromentin A, et al: Inconstant association between 27-kDaheat-shock protein (Hsp27) content and doxorubicin resistance in human colon cancer cells. The doxorubicin-protecting effect of Hsp27. Eur J Biochem 237: 653-659, 1996.

17. Choi DH, Ha JS, Lee WH, et al: Heat shock protein 27 is associated with irinotecan resistance in human colorectal cancer cells. FEBS Lett 581: 1649-1656, 2007.

18. Tweedle EM, Khattak I, Ang CW, et al: Low molecular weight heat shock protein HSP27 is a prognostic indicator in rectal cancer but not colon cancer. Gut 59: 1501-1510, 2010.

19. Yu Z, Zhi J, Peng X, Zhong X and Xu A: Clinical significance of HSP27 expression in colorectal cancer. Mol Med Rep 3: 953-958, 2010.

20. Wang F, Zhang P, Shi C, Yang Y and Qin H: Immunohistochemical detection of HSP27 and hnRNP K as prognostic and predictive biomarkers for colorectal cancer. Med Oncol: Aug 23, 2011 (Epub ahead of print).

21. Tsuruta M, Nishibori $\mathrm{H}$, Hasegawa $\mathrm{H}$, et al: Heat shock protein 27 , a novel regulator of 5-fluorouracil resistance in colon cancer. Oncol Rep 20: 1165-1172, 2008.

22. Kamada M, So A, Muramaki M, Rocchi P, Beraldi E and Gleave M: Hsp27 knockdown using nucleotide-based therapies inhibit tumor growth and enhance chemotherapy in human bladder cancer cells. Mol Cancer Ther 6: 299-308, 2007.

23. Rocchi P, So A, Kojima S, et al: Heat shock protein 27 increases after androgen ablation and plays a cytoprotective role in hormonerefractory prostate cancer. Cancer Res 64: 6595-6602, 2004.

24. Rocchi P, Beraldi E, Ettinger S, et al: Increased Hsp27 after androgen ablation facilitates androgen-independent progression in prostate cancer via signal transducers and activators of transcription 3-mediated suppression of apoptosis. Cancer Res 65: 11083-11093, 2005.

25. Sun Y, Tang XM, Half E, Kuo MT and Sinicrope FA: Cyclooxygenase-2 overexpression reduces apoptotic susceptibility by inhibiting the cytochrome c-dependent apoptotic pathway in human colon cancer cells. Cancer Res 62: 6323-6328, 2002.

26. Kluck RM, Bossy-Wetzel E, Green DR and Newmeyer DD: The release of cytochrome $\mathrm{c}$ from mitochondria: a primary site for Bcl-2 regulation of apoptosis. Science 275: 1132-1136, 1997.

27. Li P, Nijhawan D, Budihardjo I, et al: Cytochrome c and dATPdependent formation of Apaf-1/caspase- 9 complex initiates an apoptotic protease cascade. Cell 91: 479-489, 1997.

28. Concannon CG, Orrenius S and Samali A: Hsp27 inhibits cytochrome c-mediated caspase activation by sequestering both pro-caspase-3 and cytochrome c. Gene Expr 9: 195-201, 2001. 